\title{
An Information Based Model of Consciousness Fully Explaining the Mind Normal/Paranormal Properties
}

\begin{abstract}
Florin Gaiseanu
\section{ABSTRACT}

The main informational components of consciousness are described as Operative Informational System (OIS) assuring the reactive short-time adaptation and Programmed Informational System (PIS), assuring the life maintenance and the species survival, working in an integrated manner with the informed matter IM (body). The defined informational subsystems allow to describe consciousness as a sum of cognition centers defined by Ibelieve, $\mathrm{I}_{\text {know, }} \mathrm{I}_{\text {want }}, \mathrm{I}_{\text {love }}, \mathrm{I}_{\mathrm{am}}, \mathrm{I}_{\text {create }}$ and $\mathrm{I}_{\text {created. }}$. The cognition center $\mathrm{I}_{\text {believe }}$ was defined as related with the anti-entropic field of the bipolar universal matter/antimatter informational system, assisting the life structuring and health and explaining the precognition, psychokinesis, near-death experiences and other "paranormal" phenomena. PIS related activity of the cognition centers was described as the projected data in OIS by two components: (1) personal status as survival needs; (2) the info-consequences on oneself perception and action impulses perceived by $I_{a m}, I_{c r e a t e}$ and $I_{c r e a t e d}$, reflecting together with $I_{k n o w} I_{\text {want }}$ and $I_{\text {love }}$ the "normal" (regular) mind properties.
\end{abstract}

Key Words: information based model, consciousness, cognition centers, normal/paranormal mind properties DOI Number: 10.14704/nq.2017.15.2.1040 NeuroQuantology 2017; 2: 132-140

\section{Introduction}

The consciousness nature remained since centuries one of the most attractive topic especially for philosophers (Perlovski, 2001), because although this is of major importance to understand the human body mechanisms, the connection of mind with the world and with the body itself, the technological development of the investigation tools was not able to allow the application of some non-invasive techniques, as it is possible today (Kaku, 2014). Even disposing now of high micro and nanotechnology performing systems, especially based on the explosive development of integration circuits and microsystems, the most researches are dedicated to the correlation between the characteristics of the low frequency electrical currents flowing through the brain circuits and the mind activity, with specific application for the life quality improvement by medical recuperation/helping tools (Kaku, 2014). Low understanding is available yet concerning the mind support nature and mind/body relation, in spite of quite reaches literature referring to such a topic (Smith, 2001; Radin, 2006; Derek \& Arun, 2008). Notable progresses are to be remarked as concerns the quantum proposed model involving the participation of the so called microtubules, acting as high frequency oscillators, identified in the brain neurons (Hameroff, 1998; Hameroff \& Penrose, 1996, 2014). However, although some information-based proposals were suggested earlier (Chalmer, 1995; van Lommel, 2006; Walker, 2013), only recently there

\footnotetext{
Corresponding author: Florin Gaiseanu

Address: Ph.D., Senior Researcher 1 - st Degree (Professor), Associated Member of the Academy of Romanian Scientists - Information Science and Technology, Bucharest, Romania

e-mail $\bowtie$ fgtext@yahoo.es

Relevant conflicts of interest/financial disclosures: The authors declare that the research was conducted in the absence of any commercial or financial relationships that could be construed as a potential conflict of interest.

Received: 5 March 2017; Accepted: 12 March 2017
} 
were reported the basic concepts and arguments for an information-based model of consciousness (Gaiseanu, 2016 a, b), able to explain "extra"cognitive phenomena like near-death experiences.

In this paper it is presented a detailed and coherent information based model of consciousness described in terms of its informational subsystems, showing how each subsystem is reflected by its specific task in the conscious mind, according to their specific functions. On this basis it is deduced the general informational "scheme" of consciousness, described in terms of cognition centers, fully explaining both the "normal" (regular) properties of mind in relation with the human body as reflected in the conscious zone, and the connection with the universal informational matter/antimatter field, with its consequences concerning the paranormal phenomena like premonition, mind spontaneous communication at distance (Radin, 2006), psychokinesis (Gaiseanu, 2016 a), demonstrated for instance by the mind power induced cloud dissipation (Ignatenko, 1994), the mind-generated diagnostics, premonition, consciousness disembody and retro-vision to the infancy during the clinical and near-death experiences (Gaiseanu, $2016 \mathrm{~b}$ ). It is shown also that this informational model of consciousness reflects the dual nature of universe from quantum/micro to macro-scale level and of the human body, as inseparable part of it.

\section{Information-based model of consciousness}

As it was argued earlier (Gaiseanu, 2016 a), consciousness is practically an integrated informational system able to absorb and emit information. In order to relate consciousness as a contribution of various subsystems, we have to introduce first of all the specific informational concepts. Moreover, as part of our universe, we have to observe its properties under the light of new discoveries from quantum/micro to macroscale structures, as it is presented below.

\subsection{Dual informational nature of universe and of life structure}

Starting from the previous philosophical vision on information (Draganescu, 1979, 1990, 2004), as a fundamental component of the non-living and living structures in our universe, it was shown (Gaiseanu, 2016 a) that the latter researches confirmed actually that information is one of the associated component of matter (Tagmark, 2014; Gates, 2010). According to such a conception,
Structured matter = "Deep matter" + "Informatter" and Living = Structured matter + Informatter, where "Deep matter" is a neutral category, unable to structure by itself without the intervention of "Informatter" as a phenomenological/energy-supported

contributor. The living structures are the result of an intervention of information on structures, adding to it new characteristics.

Following this line, we can observe that the variation of physical quantities is actually information that can lead and even determine the evolution of a system. If we analyze therefore a system from the dynamical point of view, as the organism is, we have to observe that information is therefore a deviation from equilibrium, which can be transmitted and causes a "tendency" of the system of which that information is part. The vectors are characterized not only by their physical size, but also by direction and sense, thus giving to information and to the system which includes it a well-defined orientation. Starting from the observation that everything in nature is subjected to well-defined mathematical laws, it was suggested (Tagmark, 2014) that removing layer after layer towards the ultimate material level, we arrive to the fundamental essence of the universe, that of laws, of building-rules of training and organization. Operating with information as an independent parameter and rewriting some fundamental equations of the elemental particles, it was possible to obtain new results concerning the structuring/codification rules in our universe (Gates, 2010). Moreover, it was possible recently to deduce the empirical Newton's gravitational (mass-attraction) law (Verlinger, 2010) using information as physical parameter.

Therefore, according to Draganescu's vision, it could be attributed to the informational component a property that can be called "intention", depending on the direction and sense with respect to a reference. Following this line, we can observe that the fluctuations around equilibrium emphases two forces, one of expansion and another one of compression. The equations in mathematics and physics are not something else than the concrete expression of various groups of parameters and defined quantities into a system, and which represent actually properties of it, so therefore information. This signifies actually no more and no less than the expression of the equilibrium between groups of opposite parameters or opposite tendencies, which are confronted each other to obtain such an equilibrium. 
The mathematical and physics equations describe the evolution of the natural phenomena into a certain system, so information on the reality itself. The competition between the sign $(+)$ and $(-$ ), these symbols representing at the conceptual (informational) level nothing else than two opposite facets, conducts to equilibrium as a final result. The equilibrium is a result therefore of opposite forces like action/reaction, positive/negative (charge, electro-magnetic force), gravity/anti-gravity, entropy/antientropy, YES/NO decision, which can be associated correspondingly to the symbolic (binary) informational unit $(1,0)$. At the microparticle level, the equilibrium could be regarded more generally as a dynamical process, represented by a reaction $A+B<=>(A B)$, which is very common in chemical and solid (semiconductor) intimate processes describing the association/dissociation mechanisms. Specifically, some complementary species, defined so by their complementary properties, could associate/dissociate to form a (neutral) new unit, according to the above reaction, as an example of informational operation. The living structures are based on such reactions, forming large organic structural chains with stable or metastable properties.

A more particular case of such a situation, which could be written as: $A+B<=>0$, is representative for the generation/recombination process like electron/hole (positive charge consisting in the lack of electron), atom/vacancy (defined as a lack of the atom in its lattice site), in semiconductors like silicon (Gaiseanu, 2013), but also the generation/recombination of matter/antimatter by quantum fluctuations. As it was recently shown, our universe should be composed by matter and antimatter (Haidukovic, 2011 a, b, 2012), where antimatter would be characterized by anti-symmetrical properties (Caroll, 2015). Matter and antimatter generated by quantum fluctuations are permanently separated (Haidukovic, 2011 a, b, 2012), forming actually an informational unit of opposing/competing components expressible in Bits (Gaiseanu, 2016 a). Following the analysis at macro-scale level, it was shown that the gravitational field is actually an entropic one (Verlinger, 2010), because it acts for the increasing of a mass entropy (like the human body) attracted to another one (like earth).

In silicon, due to the thermal energy, there are generated pairs electron/holes, which could be separated by an electrical field. In the universe, matter/anti-matter particles could also be generated by quantum fluctuations and could recombine to produce an explosive energy, or could be separated by a repulsive force. The interchange of an atom in an interstitial position in semiconductors with a vacancy (opposite "particle" in a substitutional site) leads to a new state and then to a new configuration assisted by information (configuration state change) of this system. The stable equilibrium between two opposite species according to the above relation could be written as: $A+B=0$, showing that their confrontation gives a null result, or $\mathrm{A}=-\mathrm{B}$, which is actually the defining relation between them, equivalent with the decisional informational definition YES = - NO. An inequality determines a flow to a certain direction, perceived as well at the macroscopic level, conducting/imposing a new configuration of the system.

Observing the universe at the microscopic and macroscopic scale, we are therefore in the situation to understand that for any component which produces an effect to a certain sense, it is necessary and obligatory to search the component producing the contrary effect, to obtain the equilibrium. In other words, one gives rise/determines the other. A tendency to a direction (info input), gives rise to a contrary tendency (reactive info), the two tendencies coworking/confronting each other.

A culmination of such theoretical predictions was the recent experimental discovery demonstrating that the properties of particles or systems of particles could be separated (disembodied) from their mass (Denkmayr et al., 2013; Aharonov et al., 2013), allowing therefore to refer to the set of properties as to an informational field of matter (Gaiseanu, 2016 b).

\subsection{Connection to the anti-entropic universal field}

According to such results, it is justified to deduce that:

(A) we can refer to an informational separable field of matter and antimatter (Gaiseanu, $2016 \mathrm{a}$ );

(B) the nature of universe, both at micro and macro-scale level is dual, fundamentally expressed by the matter/antimatter dualism, where the two components show anti-symmetric properties; that means the time arrow passing from present to the future and the gravitational force are associated to matter, while an antigravitational (repulsive) force (Haidukovic, 2011 
$a, b, 2012$ ) and a reversely-oriented arrow of time (Caroll, 2015) should be associated to antimatter; (C) the system matter/antimatter can be assimilated with an informational system expressible in Bits (Gaiseanu, 2016 a);

(D) information is a separable part of matter, assuring the structuring/restructuring of matter;

(E) as anti-symmetrical component of the universe, anti-matter is characterized by an antigravitational field, which is actually an antientropic one, while the gravitation field associated with matter is entropic (Verlinger, 2010), disorganizing the structures;

(F) the living entities are structured by additional information with respect to non-living matter;

(G) as part of this universe, the living structures should be connected to the bipolar matter/antimatter field of universe, with all the natural consequences (Gaiseanu, 2016 a, 2017).

The last conclusion is of a special interest for us. Indeed, observing that the living organisms are self-organizing systems, permanently working to maintain their specific structure with respect to the destructive (matter) agents of the external ambient, it is natural to observe that the antigravitational (and then anti-entropic) field-source of the universe, would be a valuable candidate/protagonist acting for the living structuration. We will refer to this connection as to an anti-entropic pole of the organism, assisting the life structuring processes and the extracognition phenomena. The consequences of the connection to this pole on the specific properties of consciousness will be deduced below.

\section{Informational model of consciousness}

The operation mode of the mind is purely informational. From the beginning, we have to observe that we receive "pure" (disembodied $=$ mass-free) information by our external and internal sensors defined in Fig. 1 by ES and IE respectively. This information is stored in, or recalled from memory and used to provide a decision, transmitted to execution elements EE (Fig.1).To operate from informational point of view with specific parameters, it is suitable to define the info-creational field as the field where the thought is operator, trapping/stoking/operating and recognizing/lecture/transmitting information as its specific function.

\subsection{Informational subsystems consciousness}

Observing from the informational perspective the informational structure of consciousness, it is to deduce that this is constituted by two main components:

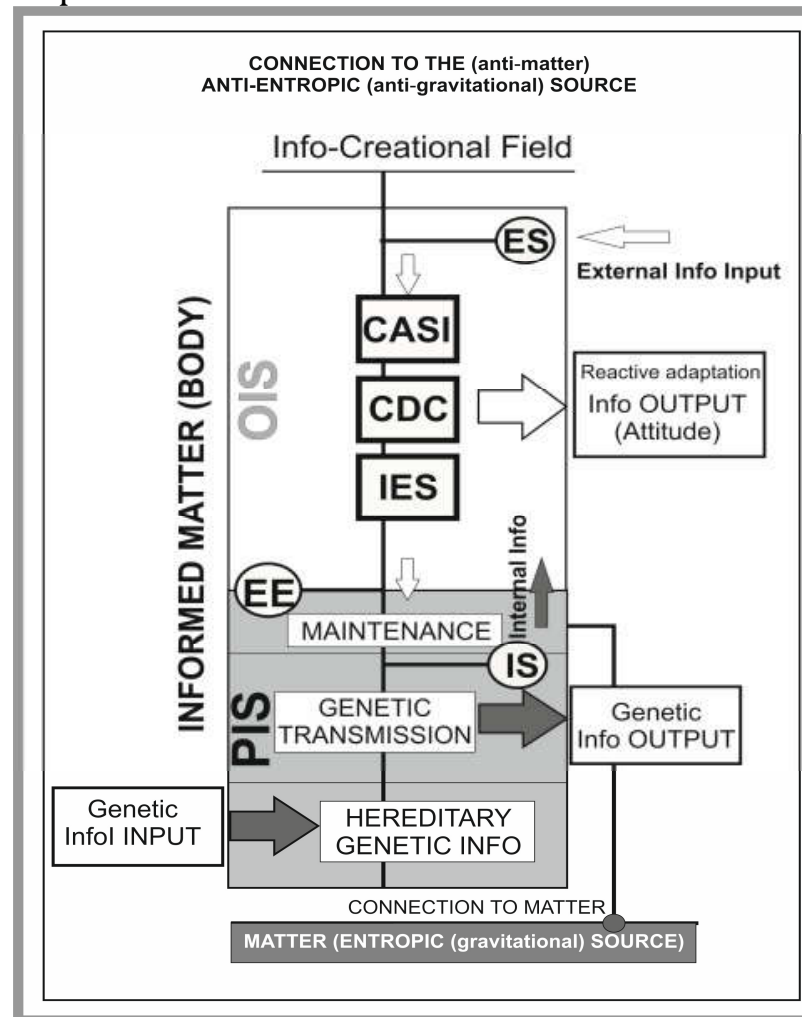

Fig. 1. Schematic representation of informational system of the human body and of its components, OIS and PIS, where OIS is the Operative Informational System composed by CASI (Center of Acquisition and Storing of Information), CDC (Center of Decision and Command) and IES (Info-Emotional Subsystem) and PIS is the Programmed Informational System. The informational system is connected to External (ES) and Internal (IS) Sensors and to the Execution Elements (EE).

(C1) the Operative Systems (OIS), able to receive information from external (ES) and internal (IS) sensors, to store it in the so called Center of Acquisition and Storing of Information (CASI), to process it in the Center of Decision and Command (CDC), to associate emotions to the initial information by Info-Emotional Subsystem (IES) and to provide a final decision as ATTITUDE, which is actually the output processed information, as it is schematically presented in Fig. 1 ;

(C2) the Programmed Informational System (PIS), able to maintain the functionality of the entire body (defined as informed matter (IM)) and to assure the survival and evolution of specie by the genetic transmission (reproduction), which is actually the info-genetic output. According to its of functions, PIS is constituted from the following components: (i) maintenance subsystem, managing the material processes dedicated to the 
body maintenance (metabolism); (ii) genetic transmission subsystem for reproduction, acting as an informational output consisting in codified information on material support; (iii) genetic information generator, acting as a hereditary input codified information inherited from parents and assuring the body development according to the age and main behavioral features and affinities.

The emotions represent the collection of informational transmitted signals as a reaction to an input information from ES or IS and collected by CASI. Emotions could be also (re)activated by the recalling of already existing (imprinted) information from CASI. Therefore, as well as the input info, the emotions are also acquired and stoked (memorized) information in CASI, as a reactive form to input info received from ES or IS, so in Fig. 1 we have to consider OIS as including the Info-Emotional Subsystem (IES).

The name of the info-creational field is derived from its own specific function, dedicated especially to detect (new) information from internal or external sources and to create new information from that existing already in CASI. A successive repetitive process as an information chain determines its conversion in an automatic acquired routine, which will be added to PIS as a behavioral acquisition.

\subsection{Cognition centers of consciousness}

According to the presented scheme in Fig. 1, consciousness is actually an informational system, receiving/processing/returning information from/to the environment and from/to itself, operating fundamentally in two main manners:

- operatively, responding to the environmental conditions for short-time adaptation/survival by the ATTITUDE, as information output;

- automatically, acting to maintain its own configuration and assuring the reproduction, as a long-time adaptation/survival informational output.

At level of personal perception, OIS could be associated with the conscious mind, serving for the connection with the environment and the adaptive immediate reaction response to the informational stimuli. Therefore, OIS is directly connected to the basic information sensors. PIS is devoted basically to the maintenance of the vital functions of the body and to the furnishing of stable/checked/accepted/integrated genetic information to OIS and to output, so this would act as the subconscious zone of the consciousness.
Defining our own perception on these systems (Fig. 1) as cognition centers (Fig. 2), we have to observe that the personal perception of CASI, our data library, could be suggestively

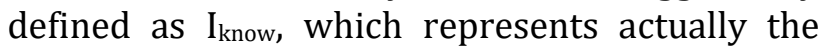
informational experience of I. In the same manner, the output information delivered by CDC should be defined as $\mathrm{I}_{\text {want }}$ and in a similar way we can refer to IES as to a personalized emotional I, which can be named $\mathrm{I}_{\text {love }}$, because it is naturally set to love, to enjoy the life. Taking into account its main function of maintenance of body, the zone of PIS responsible for this function would represent actually the part of I which could be defined by $\mathrm{I}_{\mathrm{am}}$. We can define also the function of PIS dedicated to the genetic transmission from the parents by $I_{\text {created, }}$ because it assures the genetic development and evolution of the body, according to the genetic specific program. Accordingly, $I_{\text {create }}$ would represent the genetic transmission output information, described by the long-term adaptation/evolution circuit for survival in the informational model represented in Fig. 1.

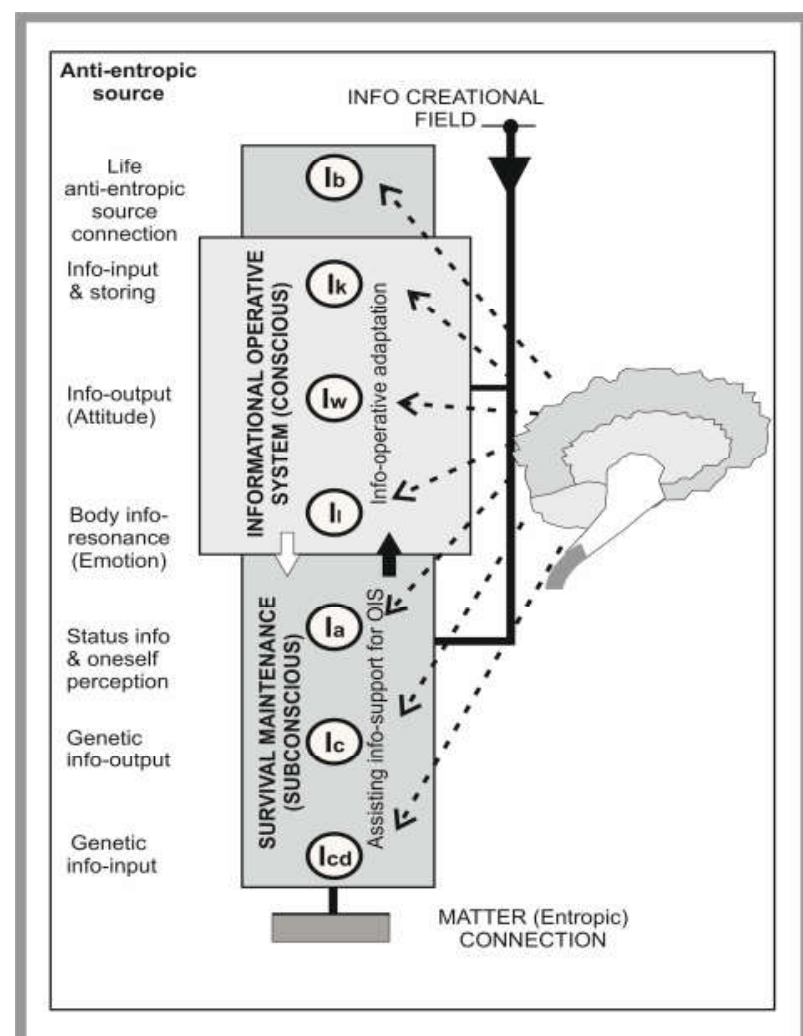

Fig. 2. Schematic representation of the cognition centers, connected to the info-creation field and matter: Ibelieve $->\mathrm{I} b$, $\mathrm{I}_{\text {know }} \rightarrow \mathrm{I}_{\mathrm{k}} \rightarrow \mathrm{I}_{\text {want }} \rightarrow \mathrm{I}_{\mathrm{w}}$, Ilove $>\mathrm{I}_{\mathrm{l}}$, Iam $\rightarrow \mathrm{I}_{\mathrm{a}}$, Icreate $>\mathrm{I}_{\mathrm{c}}$ and $\mathrm{I}_{\text {created }}->\mathrm{I}_{\mathrm{cd}}$.

As it is shown in Fig. 1, PIS is connected directly to matter, actually by means of $\mathrm{I}_{\mathrm{am}}$, dedicated to assign the necessary material for the construction/restructuring processes and maintenance of the organism. This could be 
considered therefore as a pole of connection with matter. The other side of bipolar connection of the organism would be represented by the infocreational field, connected to the anti-entropic field of antimatter (Fig. 1), felt normally as a stability/benevolence/safety pole of the life, which we can define as $I_{\text {believe. The bipolar feature }}$ of universe is therefore reflected at individual level by the binomial unit spirit/matter, where

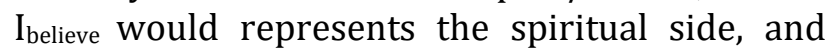
matter the material side.

The cognitive I could be therefore regarded as the sum of the contribution of all I subsystems defined above (Fig. 2), under the form: $I=I_{\text {created }}+I_{\text {create }}+I_{\text {am }}+I_{\text {love }}+I_{\text {want }}+I_{\text {know }}+I_{\text {believe }}$ (1) For simplicity we will refer to these components as follows: $I_{\text {believe }}>>I_{b}, I_{\text {know }}>I_{k}, \rightarrow I_{\text {want }}->I_{w}, I_{\text {love }}-$ $>\mathrm{I}_{\mathrm{l}}, \mathrm{I}_{\mathrm{am}}>>\mathrm{I}_{\mathrm{a}}, \mathrm{I}_{\text {create }}>>\mathrm{I}_{\mathrm{c}}$ and $\mathrm{I}_{\text {created }}>>\mathrm{I}_{\mathrm{cd}}$, as it also indicated in Fig. 2.

\section{Normal/paranormal properties of mind}

Consciousness is an integrated system of the human body, implying a close relation and an interchange of information both between the subsystems and the body itself (Gaiseanu, 2016 b), so we have to discuss in more detail these relations, as presented below.

\subsection{The normal (regular) properties of mind}

The normal properties of mind could be deduced analyzing the specific properties of the cognition centers as defined above. A full description of the cognition centers $I_{a m}, I_{\text {create }}$ and $I_{\text {created }}$ specific to PIS, dedicated especially to programmed/ codified information processing, should include actually the projected data in the conscious zone, as primary defined function. We have to note also the following: as $I_{a m}$ derives from the main function of PIS referring to the maintenance of body by connection with matter (air, foods, water), this is from this point of view a matterrelated center. The subsystems $\mathrm{I}_{\text {create }}$ and $\mathrm{I}_{\text {created }}$ are also included in such a category, because $\mathrm{I}_{\text {create }}$ is dedicated to conduct the genetic transmission system, while I $_{\text {created }}$ to develop the evolution of the body according to the age and the genetic transmission from the parents. The correlation between the "matter-related" subsystems specific to PIS (unconscious zone) and the "info-related" subsystems (included in OIS as conscious zone) will be presented as below, starting as a concrete example from the analysis of the subsystem $\mathrm{I}_{\mathrm{am}}$.

This subsystem consists in two kinds of components: (1) automatically working algorithms related to the body maintenance, which we define as info-programmed algorithms (IPA); (2) info-projection of IPA in the operative informational system (OIS), defined as infoprojected data (IPD). Taking into account these definitions, we can write the following relation:

$\mathrm{I}_{\mathrm{am}}=\mathrm{I}_{\mathrm{am}}$ (IPA) $+\mathrm{I}_{\mathrm{am}}$ (IPD)

To be more explicit, we have to note that IPA represents actually in this case the infomaintenance activity of the body dedicated mainly to the matter absorption/desorption processing, necessary to assure the energy and the recuperating material for IM. On the other hand, IPD includes the following informational processes: (1) the info-reception signals coming from PIS by means of IS, concerning the matterenergy related survival needs (hunger, thirst, temperature, pain); (2) the info-personal perception on oneself, which is stoked actually in CASI $\left(=I_{k}\right)$. Taking into account this specification, we can write the relation:

$$
\begin{aligned}
& \mathrm{I}_{\mathrm{am}}(\mathrm{IPD})=\mathrm{I}_{\mathrm{am}} \text { (matter-related status) }+\mathrm{I}_{\mathrm{am}} \\
& \text { (oneself perception) }
\end{aligned}
$$

Where the matter-related status signifies the set of needs specified in (1), while the oneself perception is represented by (2). Therefore, $I_{a m}$ (IPD) is a provider of information on matterrelated status and oneself-related perception.

On the same way, we may define $I_{\text {create }}$ (IPA), expressing the info-procreation process in PIS, necessary to the info-genetic transmission (as an output genetic (codified) information), and $I_{\text {create }}$ (IPD), expressing the info-signals perceived by OIS, described as follows: (1) by infoprocreation status signals received by means of IS from PIS; (2) by sociability and info-creativity features as info-related characteristic impulses. Therefore, as in the precedent case, we can write the following relation:

$I_{\text {create }}=I_{\text {create }}\left(\right.$ IPA) $+I_{\text {create }}$ (IPD)

Where I $_{\text {create }}$ (IPA) represents actually the infooutput by genetic transmission of the long-time adaptation/survival circuits, as shown in Fig. 1, and

$I_{\text {create }}\left(\right.$ IPD) $=I_{\text {create }}$ (procreation status) $+I_{\text {create }}$ (sociability/creativity)

Where the procreation status represents the item (1) in the above description, while sociability/creativity represents the description from item (2). Therefore, we have to observe that $I_{\text {create }}$ is a provider of procreation and sociability/creativity info.

Following the same kind of reasoning, we can write that:

$I_{\text {created }}=I_{\text {created }}(I P A)+I_{\text {created }}(I P D)$ 
Where $\mathrm{I}_{\text {created }}$ (IPA) is an injector of genetic (codified) info obtained from parents, acting for the development and evolution of body from the first moments of the conception to death, and $I_{\text {created }}$ (IPD) is expressed by (genetic) predispositions, principles/morality/ (selection criteria) genetically acquired or learned from parents during the first years of life and transmitted to OIS, serving for the decision process. Therefore, as in the previous cases we can write:

$$
\mathrm{I}_{\text {created }}(\mathrm{IPD})=\mathrm{I}_{\text {created }}(\text { predispositions })+\mathrm{I}_{\text {created }}
$$
(selection criteria)

As OIS is dedicated especially to information related processes (inforeception/decision/transmission), $\mathrm{I}_{\text {love }}$ is a provider of info-emotional experience, $\mathrm{I}_{\text {know }}$ represents the informational experience and $I_{\text {want }}$ the decision as the output information of the short-time adaptation circuit. The subsystem $\mathrm{I}_{\text {believe }}$ is the provider of certitude and life/objective security and high aspirations, this being connected to the anti-entropic source of the organism.

The subsystems composing OIS transmit command signals to the execution elements EE by means of $\mathrm{I}_{\text {want. }}$. If the decision signals are frequently repetitive, this will be assimilated in PIS as a new (acquired) programmed subroutines, becoming a stereotype behavior to be used for the typical situations.

\subsection{The paranormal properties of mind}

The so called paranormal properties of mind, only poor or at all explained up today, could be deduced as a consequence of the exposed informational model. For this, we have to take into account that the connection pole to the anti-entropic field is part of our complex informational system. Therefore, the effects of this component on consciousness have to be taken into account to completely understand the complex phenomena associated to it. The connection to the antientropic field as an opposite/structuring force against the entropic/destructive field of matter is represented in Fig. 1 as an anti-entropic pole of the organism. More explicitly, while the maintenance subsystem controls the interaction with matter, the connection with anti-entropic field acts as a structuring/assisting force of the organism, with subtle extra-effects on the consciousness, perceived as "paranormal" phenomena. That is because as it was commented above and was argued earlier (Gaiseanu, 2016 a), the infocreational field is connected to the anti-entropic (anti-gravitational) field of anti-matter, which forms together with the entropic (gravitational) field of matter an universal informational field, assisting the life structuring and its maintenance.

Therefore, following important characteristics are of main interest for our discussion, as deduced from the informational model of consciousness: (i) the info-creational field is connected to the informational universal unit of our universe, consisting in the entropic (gravitational)/anti-entropic (anti-gravitational) field; (ii) the anti-gravitational field acts as an antientropic source, assisting the life structuration and then the health of the human body; (iii) the anti-gravitational field of anti-matter acts against the gravitation force; (iv) the anti-gravitational component of the universe is reversely timeoriented, namely from the future to the present, according to the conclusion (B).

The following consequences on the properties (evidences) experimentally observed could be therefore deduced:

(1) the mind could communicate "spontaneously" by means of the info-creational field; this is possible due to the dualism of matter/antimatter field forwardly/reversely time-oriented, giving to such a communication the character of "spontaneous"; in other words, under special conditions and for compatible (let's say „entangled") minds, an emitted "info-clip" within the info-creational field by a person, will be perceived coming from the future to present by another one, then perceived "spontaneously", by means of the anti-entropic pole of the universe; (2) in the same way could be explained the premonition phenomenon, which consists in the perception of an event (coming actually to the receiver by means of the anti-entropic field) before it would take place in the matter-related field where we really live; that is because the time is reversely oriented in in the anti-entropic field with respect to the regular matter field of our regular (normal) field;

(3) the extra-corporal view is explained also by means of info-creational field: the normal (direct) view by means of the ES (eye) sensor, is substituted by the "paranormal" virtue of the mind, connected to the informational field of consciousness, which may absorbs/contains/transmits informational infoclip messages; the mind obtains under such conditions an "observable" internal access (as "internal view") to information supported by the informational field, "scanning" it directly; 
(4) the practice of the internal view allows to the clairvoyants the "scanning" of the corporal informational structure and the detection of its functional/structural deviations from the normal configuration/work, allowing valid health diagnostics (Myss, 1996);

(5) the near-death experiences consisting in detachment (described by various experimenters as an absorption in an imponderable, fine-world, where the laws of physics are different of the known ones), extra-corporal view (described above), the exploration/judgment of oneself life retrospectively (an effect of the reverselyoriented time arrow and the resetting to the "haven" (perfect) informational structuring laws of life), disembody (information separation from informed matter (body), which becomes a regular (ordinary) matter), and the tube (funnel) absorption as a consequence of disembody (Gaiseanu, 2017);

(6) the psychokinetic phenomena, as for instance the cloud dissipation by the mind (Ignatenco, 1994) and levitation could be explained by the connection to the anti-entropic and antigravitational field (conclusion (E)) (Gaiseanu $2016 \mathrm{a}$ )) and its amplification by the mind control.

\section{Conclusions}

1. The consciousness could be described as an integrated informational system, receiving and transmitting information between its components and from/to the environment in order to maintain with it a dynamic equilibrium, allowing its short and long-term adaptation/survival.

2. The informational model of consciousness based on adaptation/survival subsystems OIS and PIS and their components, allows defining the informational cognition centers.

3. The Operative Information System is perceived as the conscious mind and the Programmed Informational System as the subconscious mind, automatically working.

3. The perceived informational subsystems can be described in terms of $\mathrm{I}$, defined as cognition centers $I_{\text {believe, }}, I_{\text {know }}, I_{\text {want }}, I_{\text {love }}, I_{a m}, I_{\text {create }}, I_{\text {created, }}$ where $I_{\text {know }}, I_{\text {want }}$ and $I_{\text {love }}$ are related to the components of OIS, implied mainly in freely infoprocessing, while $I_{a m}, I_{\text {create, }} I_{\text {created }}$ to PIS components, leaded by codified/programmed information.

4. Ibelieve was defined as a cognition subsystem connected to the info-(anti-entropic) universal source, assisting the life structuring, its maintenance and healthy.
5. $\mathrm{I}_{\mathrm{i}}(\mathrm{i}=\mathrm{b}, \mathrm{k}, \mathrm{w})$ are mainly informational-type subsystems, because refer to the reception/processing/transmission of information; particularly, $I_{w}$ has the role to transmit to the execution elements the final decision, as output information.

6. The specific characteristics of PIS related components $\left(\mathrm{I}_{\mathrm{am}}, \mathrm{I}_{\text {create }}\right.$ and $\left.\mathrm{I}_{\text {created }}\right)$ were described by their main function in PIS and by the corresponding projection in the conscious mind (OIS) according to the general relation: $\mathrm{I}_{\mathrm{i}}=\mathrm{I}_{\mathrm{i}}$ (IPA) $+\mathrm{I}_{\mathrm{i}}$ (IPD) where $\mathrm{i}=\mathrm{a}, \mathrm{c}, \mathrm{cd}$, IPA represents the infoprogrammed algorithms and IPD info-projected data in OIS.

7. The subsystems $I_{i}$ (IPD) $(i=a, c, c d)$ were described by two components: (1) personal status perceived by signals coming from PIS to OIS, consisting mainly in matter/energy-related survival needs (hunger, thirst, temperature, pain, procreation, or other genetic-origin impulses); (2) the info-consequences of needs on oneself perspectives (oneself view, sociability/creativity, selection criteria).

8. The defined/observed/detected features of the cognition centers allow to explain the normal (regular) properties of consciousness as described above;

(9) The special class of phenomena considered/known as a paranormal category, poorly or at all understood up to date, can be explained in terms of the informational model, showing that the mind is connected to the informational anti-gravitational (anti-entropic) field of anti-matter with anti-symetrical properties with respect to that of matter;

(10) The informational model is able to support/explain the spontaneous ("entangled") mind communication, premonition, extracorporal view, illness/dysfunctional diagnostics by mind exploration of informational field, neardeath experiences of disembody, detachment, oneself-retrovision and resetting to the perfect physics laws of life, funnel absorption, psychokinesis and levitation phenomena.

(11) The matter/antimatter dualism of our universe is reflected in consciousness as normal/paranormal properties.

The proposed informational model of consciousness fully explaining the normal and paranormal properties of mind opens new perspectives for the investigation of the nature of consciousness, its internal and external connections and the intimate relations lifeuniverse. 
NeuroQuantology | June 2017 | Volume 15 | Issue 2 | Page 132-140 | 10.14704/nq.2017.15.2.1040

Gaiseanu F., An Information Based Model of Consciousness Fully Explaining the Mind Normal/Paranormal Properties

\section{References}

Aharonov Y, Popescu S, Rohrlich D and Skrzypczyk P. Quantum Cheshire Cats, New Journal of Volume Physics 2013; 15:1-5. http_iopscience.iop.org_1367_2630_15_11_113015_art.p df.

Carroll S. The Arrow of Time. www.proposterousuniverse.com and From Eternity to Here, the Quest for the Ultimate Theory of Time, GB: Oneworld Publication, 2015.

Chalmers D. Facing Up to the Problem of Consciousness. Journal of Consciousness Studies 1995; 2 (3): 200-219.

Denkmayr T., Geppert H., Sponar S., Lemmel H., Matzkin A., Tollaksen J. Observation of a quantum Cheshire Cat in a matter wave interferometer experiment 2013. http://www.arxiv.org/pdf/1312.3775, Dec. 1.

Derek A., Paul D., Arun C. P., Editors Quantum Aspects of Life, forword by Sir Roger Penrose 2008; Imperial College Press.

Draganescu M. The Depths of the Material World (in Romanian), Bucharest: Editura Politica 1979. The Depth of Existence (translation in English) Bucharest: Ed Pol.1997. http://www.racai.ro/books/doe.

Draganescu M. Information of Matter (in Romanian). 1990; Bucharest: Romanian Academy.

Draganescu M. The Fundamental Phenomenological Information of the Universe. Proc. of the Romanian Academy, Series A 2004; 5 (1): 51- 58.

Gaiseanu F. Contributions to the Modelling and Simulation of the Atomic Transport Processes in Silicon and Polysilicon and Applications, Proceedings of the Romanian Academy, Series A, The Publishing House of the Romanian Academy 2013; 4(4): 376-384. (http://www.acad.ro/sectii2002/proceedings/doc20134/15-Gaiseanu.pdf) .

Gaiseanu F. Consciousness as Informational System of the Human Body. Consciousness and Life Physics, Cosmology and Astrophysics Journal 2016 a; 16 (1): 14-25.

Gaiseanu F. Informational Subsystems of the Consciousness. The Science of Consciousness, Abstract Book, TSC 2016 TUCSON, edited by The University of Arizona Center for Consciousness Studies and University of Michigan Center for Consciousness Science, by Stuart Hameroff (The University of Arizona) and George Mashour (University of Michigan), 2016 b; April 25-30, 9: 29. http://www.consciousness.arizona.edu/documents/TSC 2016_BOOK_of_Abstracts_for_emailWeb.pdf.

Gaiseanu F. Quantum-assisted process of disembody under near-death conditions: an informational-field support model. NeuroQuantology 2017; 15(1): 4-9. http://www.neuroquantology.com/index.php/journal/a rticle/view/971
Gates Jr. S.J. Symbols of Power. Physics World 2010; 23: 3439.

Hajdukovic D. S. Black Holes and Gravitational Properties of Antimatter. Advanced Studies in Theoretical Physics 2011 a; 5: 2-17.

Hajdukovic D. S. Is dark matter an illusion created by the gravitational polarization of the quantum vacuum?. Astrophys.Space Sci. 2011b; 334: 215-218.

Hajdukovic D. S. Quantum vacuum and dark matter. Astrophys. Space Sci. 2012; 337 (6): 9-14.

Hameroff S. Quantum Computation in Brain Microtubules? The Pennrose - Hameroff". Orch OR" Model of Consciousness. Philos. Trans. Royal Society London, Ser.A, Math. Phys. Sci. 1998; 356: 1869-1898.

Hameroff S., Penrose R. Consciousness in the Universe: a Review of the 'Orch. OR' Theory. Physics of the Life Review 2014; 11: 39-79.

Hameroff S., Penrose R. Orchestred Reduction of Quantum Coherence in Brain Microtubules: a Model for Consciousness. Math. Comput. Simul 1996; 40: 453-480.

Kaku M. The Future of the Mind, The Scientific Quest to Understand Enhance and Empowered the Mind, NY, London, Toronto, Sidney Aukland: Doubleday, Division of Random House Canada, Toronto, Penguin Random Hose Companies 2014.

Ignatenko A. How to become a phenomenon (in Romanian), Bucharest: Biodova 1994. http://www.professorignatenko.com/en/about-author.

Myss C. Ph. D., Anatomy of the Spirit - The Seven Stages of Power and Healing, USA: Crown Publishers, Inc., 1996.

Radin D. Entangled Minds, Extrasensory Experiences in a Quantum Theory. NY, London, Toronto, Sydney: Paraview Pocket Books, a division of Simon Shuster, Inc., 2006.

Perlovski L. Neural Networks and Intellect: Using Model Based Concepts. Oxford: University Press, 2001.

Smith S. W. The Inner Light Theory of Consciousness. USA: California Technical Publishing, 2001.

Tegmark M. Our Mathematical Universe: My Quest for the Ultimate Nature of Reality. USA: Randomhouse, 2014.

Verlinger E. On the Origin of Gravity and the Laws of Newton. University of Amsterdam Valckenierstraat 2010; 65: 1018 XE, Amsterdam The Netherlands, Institute for Theoretical Physics. http://www.arXiv:1001.0785v1 [hep-th].

van Lommel P. Near-death experience, consciousness, and the brain: A new concept about the continuity of our consciousness based on recent scientific research on near-death experience in survivors of cardiac arrest,World Futures 2006; 62: 134-151.

Walker SI, Davies PCW. The Algorithmic Origins of Life, Journal Royal Society Interface; 2012: 10 no. 79 20120869. https://arxiv.org/format/1207.4803. 